\title{
Real Hypersurfaces of Nonflat Complex Projective Planes Whose Jacobi Structure Operator Satisfies a Generalized Commutative Condition
}

\author{
Theocharis Theofanidis \\ School of Second Chance Delta, 57008 Thessaloniki, Greece \\ Correspondence should be addressed to Theocharis Theofanidis; onslaught5000@hotmail.com
}

Received 20 May 2016; Accepted 29 June 2016

Academic Editor: Luc Vrancken

Copyright (C) 2016 Theocharis Theofanidis. This is an open access article distributed under the Creative Commons Attribution License, which permits unrestricted use, distribution, and reproduction in any medium, provided the original work is properly cited.

Real hypersurfaces satisfying the condition $\phi l=l \phi(l=R(\cdot, \xi) \xi)$ have been studied by many authors under at least one more condition, since the class of these hypersurfaces is quite tough to be classified. The aim of the present paper is the classification of real hypersurfaces in complex projective plane $\mathbb{C} P^{2}$ satisfying a generalization of $\phi l=l \phi$ under an additional restriction on a specific function.

\section{Introduction}

An $n$-dimensional Kaehlerian manifold of constant holomorphic sectional curvature $c$ is called complex space form, which is denoted by $M_{n}(c)$. A complete and simply connected complex space form is a projective space $\mathbb{C} P^{n}$ if $c>0$, a hyperbolic space $\mathbb{C} H^{n}$ if $c<0$, or a Euclidean space $\mathbb{C}^{n}$ if $c=0$. The induced almost contact metric structure of a real hypersurface $M$ of $M_{n}(c)$ will be denoted by $(\phi, \xi, \eta, g)$.

Real hypersurfaces in $\mathbb{C} P^{n}$ which are homogeneous were classified by Takagi [1]. The same author classified real hypersurfaces in $\mathbb{C} P^{n}$, with constant principal curvatures in [2], but only when the number $g$ of distinct principal curvatures satisfies $g=3$. Kimura showed in [3] that if a Hopf real hypersurface $M$ in $\mathbb{C} P^{n}$ has constant principal curvatures, then the number of distinct principal curvatures of $M$ is 2,3, or 5. Berndt gave the equivalent result for Hopf hypersurfaces in $\mathbb{C} H^{n}$ [4], where he divided real hypersurfaces into four model spaces, named $A_{0}, A_{1}, A_{2}$, and $B$. Real hypersurfaces of types $A_{1}$ and $A_{2}$ in $\mathbb{C} P^{n}$ and of types $A_{0}, A_{1}$, and $A_{2}$ in $\mathbb{C} H^{n}$ are said to be hypersurfaces of type $A$ for simplicity. Another class of real hypersurfaces that appears quite often is the Hopf hypersurfaces where the structure vector field is a principal vector field. For more details and examples on real hypersurfaces of type $A$ and Hopf, we refer to [5].
A Jacobi field along geodesics of a given Riemannian manifold $(M, g)$ plays an important role in the study of differential geometry. It satisfies a well-known differential equation which inspires Jacobi operators. For any vector field $X$, the Jacobi operator is defined by $R_{X}: R_{X}(Y)=R(Y, X) X$, where $R$ denotes the curvature tensor and $Y$ is a vector field on $M . R_{X}$ is a self-adjoint endomorphism in the tangent space of $M$ and is related to the Jacobi differential equation, which is given by $\nabla_{\hat{\gamma}}\left(\nabla_{\hat{\gamma}} Y\right)+R(Y, \hat{\gamma}) \hat{\gamma}=0$ along a geodesic $\gamma$ on $M$, where $\hat{\gamma}$ denotes the velocity vector along $\gamma$ on $M$.

In a real hypersurface $M$ of a complex space form $M_{n}(c)$, $c \neq 0$, the Jacobi operator on $M$ with respect to the structure vector field $\xi$ is called the structure Jacobi operator and is denoted by $R_{\xi}(X)=R(X, \xi) \xi=l X$.

Real hypersurfaces have been studied from many points of view. Certain authors have studied real hypersurfaces under conditions which include the operator $l$ [6-9]. Other authors have studied real hypersurfaces under the condition $\phi l=l \phi$, equipped with one or two additional conditions [1015], proving that these hypersurfaces are Hopf and classifying them as type $A$.

In the present paper we classify real hypersurfaces of complex projective planes, satisfying

$$
(\phi l-l \phi) X=\psi(X) l X,
$$


restricted in the subspace $\mathbb{D}=\operatorname{ker}(\eta)$ of $T_{p} M$ for every point $p \in M$, where $\operatorname{ker}(\eta)$ consists of all vector fields orthogonal to the Reeb flow vector field $\xi$ and the form $\psi$ is assumed to be nonlinear with respect to scalar product. If $\psi$ is linear, then by replacing $X$ with $2 X$, we obtain $(\phi l-l \phi) X=2 \psi(X) l X$ which implies $\psi(X) l X=0$. So (1) takes the simpler form $\phi l=l \phi$.

Since this class is rather difficult to classify, a second condition is imposed. However it is not a condition acting in vector fields, but only in the function $\alpha=g(A \xi, \xi): \nabla_{\xi} \xi \cdot \alpha=$ 0 , where $A$ is the shape operator. Geometrically speaking, we demand the function $\alpha$ to be constant in the direction of the integral curves of $\xi$. Namely, we prove the following.

Main Theorem. A real hypersurface $M$ of a complex projective plane $\mathbb{C} P^{2}$, satisfying $(\phi l-l \phi) X=\psi(X) l X, \forall X \in \mathbb{D}(\psi$ is nonlinear), and $\nabla_{\xi} \xi \cdot \alpha=0$, is Hopf. Furthermore, if $\alpha=$ $g(A \xi, \xi) \neq 0$ then $M$ is locally congruent to a model space of type $A$ and $\psi(X) l X=0$.

For the case of $\mathbb{C} P^{n}$ in order to determine real hypersurface of type $A$, the technical assumption $\eta(A \xi) \neq 0$ is needed. Actually, there is a nonhomogeneous tube with $A \xi=0$ (of radius $\pi / 4$ ) over a certain Kaehler submanifold in $\mathbb{C} P^{n}$, when its focal map has constant rank on $M$ [16].

\section{Preliminaries}

Let $M_{n}$ be a Kaehlerian manifold of real dimension $2 n$, equipped with an almost complex structure $J$ and a Hermitian metric tensor $G$. Then for any vector fields $X$ and $Y$ on $M_{n}(c)$, the following relations hold:

$$
\begin{aligned}
J^{2} X & =-X, \\
G(J X, J Y) & =G(X, Y), \\
\widetilde{\nabla} J & =0,
\end{aligned}
$$

where $\widetilde{\nabla}$ denotes the Riemannian connection of $G$ of $M_{n}$.

Now, let $M_{2 n-1}$ be a real $(2 n-1)$-dimensional hypersurface of $M_{n}(c)$, and denote by $N$ a unit normal vector field on a neighborhood of a point in $M_{2 n-1}$ (from now on we will write $M$ instead of $\left.M_{2 n-1}\right)$. For any vector field $X$ tangent to $M$ we have $J X=\phi X+\eta(X) N$, where $\phi X$ is the tangent component of $J X, \eta(X) N$ is the normal component, and

$$
\begin{gathered}
\xi=-J N, \\
\eta(X)=g(X, \xi), \\
g=\left.G\right|_{M} .
\end{gathered}
$$

By properties of the almost complex structure $J$ and the definitions of $\eta$ and $g$, the following relations hold [17]:

$$
\begin{aligned}
\phi^{2} & =-I+\eta \otimes \xi, \\
\eta \circ \phi & =0, \\
\phi \xi & =0, \\
\eta(\xi) & =1,
\end{aligned}
$$

$$
\begin{aligned}
g(\phi X, \phi Y) & =g(X, Y)-\eta(X) \eta(Y), \\
g(X, \phi Y) & =-g(\phi X, Y) .
\end{aligned}
$$

The above relations define an almost contact metric structure on $M$ which is denoted by $(\phi, \xi, g, \eta)$. By virtue of this structure, we can define a local orthonormal basis $\left\{e_{1}, e_{2}, \ldots, e_{n-1}, \phi e_{1}, \phi e_{2}, \ldots, \phi e_{n-1}, \xi\right\}$, called $\phi$-basis. Furthermore, let $A$ be the shape operator in the direction of $N$, and denote by $\nabla$ the Riemannian connection of $g$ on $M$. Then $A$ is symmetric and the following equations are satisfied:

$$
\begin{aligned}
\nabla_{X} \xi & =\phi A X \\
\left(\nabla_{X} \phi\right) Y & =\eta(Y) A X-g(A X, Y) \xi .
\end{aligned}
$$

As the ambient space $M_{n}(c)$ is of constant holomorphic sectional curvature $c$, the equations of Gauss and Codazzi are, respectively, given by

$$
\begin{aligned}
& R(X, Y) Z=\frac{c}{4}[g(Y, Z) X-g(X, Z) Y \\
& \quad+g(\phi Y, Z) \phi X-g(\phi X, Z) \phi Y-2 g(\phi X, Y) \phi Z] \\
& \quad+g(A Y, Z) A X-g(A X, Z) A Y, \\
& \left(\nabla_{X} A\right) Y-\left(\nabla_{Y} A\right) X=\frac{c}{4}[\eta(X) \phi Y-\eta(Y) \phi X \\
& \quad-2 g(\phi X, Y) \xi] .
\end{aligned}
$$

The tangent space $T_{p} M$, for every point $p \in M$, is decomposed as follows:

$$
T_{p} M=\operatorname{ker}(\eta)^{\perp} \oplus \operatorname{ker}(\eta)
$$

where $\operatorname{ker}(\eta)^{\perp}=\operatorname{span}\{\xi\}$ and $\operatorname{ker}(\eta)$ is defined as follows:

$$
\operatorname{ker}(\eta)=\left\{X \in T_{p} M: \eta(X)=0\right\} .
$$

Based on the above decomposition, by virtue of (6), we decompose the vector field $A \xi$ in the following way:

$$
A \xi=\alpha \xi+\beta U,
$$

where $\beta=\left|\phi \nabla_{\xi} \xi\right|$ and $U=-(1 / \beta) \phi \nabla_{\xi} \xi \in \operatorname{ker}(\eta)$, provided that $\beta \neq 0$.

As stated before, if the vector field $\xi$ is a principal vector field, the real hypersurface is called Hopf hypersurface. In this case the vector field $A \xi$ is expressed as $A \xi=\alpha \xi, \alpha=$ $g(A \xi, \xi)$.

Finally, differentiation of a function $f$ along a vector field $X$ will be denoted by $(X f)$. All manifolds of this paper are assumed to be connected and of class $C^{\infty}$.

\section{Auxiliary Relations}

In the study of real hypersurfaces of a complex space form $M_{n}(c), c \neq 0$, it is a crucial condition that the structure vector field $\xi$ is principal. The purpose of this paragraph is to establish relations that will help us prove this condition. 
Let $\mathcal{N}=\{p \in M: \beta \neq 0$ in a neighborhood of $p\}$. If we had at least one point of $\mathcal{N}$, where $\alpha=0$, then from (7) we would obtain $l U=\left(c / 4-\beta^{2}\right) U$ and $l \phi U=(c / 4) \phi U$. Combining the last two equations with (1) we would take $\beta=0$ which is a contradiction. Therefore $\alpha \neq 0$ in $\mathcal{N}$.

Lemma 1. Let $M$ be a real hypersurface of a complex projective plane $\mathbb{C} P^{2}$, satisfying (1). Then the following relations hold in $\mathcal{N}$ :

$$
\begin{aligned}
A U & =\left(\frac{\gamma}{\alpha}-\frac{c}{4 \alpha}+\frac{\beta^{2}}{\alpha}\right) U+\beta \xi, \\
A \phi U & =\left(\frac{\gamma}{\alpha}-\frac{c}{4 \alpha}\right) \phi U, \\
\nabla_{\xi} \xi & =\beta \phi U, \\
\nabla_{U} \xi & =\left(\frac{\gamma}{\alpha}-\frac{c}{4 \alpha}+\frac{\beta^{2}}{\alpha}\right) \phi U, \\
\nabla_{\phi U} \xi & =\left(\frac{c}{4 \alpha}-\frac{\gamma}{\alpha}\right) U, \\
\nabla_{\xi} U & =\kappa_{1} \phi U, \\
\nabla_{U} U & =\kappa_{2} \phi U, \\
\nabla_{\phi U} U & =\kappa_{3} \phi U+\left(\frac{\gamma}{\alpha}-\frac{c}{4 \alpha}\right) \xi, \\
\nabla_{\xi} \phi U & =-\kappa_{1} U-\beta \xi, \\
\nabla_{U} \phi U & =-\kappa_{2} U-\left(\frac{\gamma}{\alpha}-\frac{c}{4 \alpha}+\frac{\beta^{2}}{\alpha}\right) \xi, \\
\nabla_{\phi U} \phi U & =-\kappa_{3} U,
\end{aligned}
$$

where $\kappa_{1}, \kappa_{2}$, and $\kappa_{3}$ are smooth functions in $\mathcal{N}$.

Proof. From (7) we get

$$
l X=\frac{c}{4}[X-\eta(X) \xi]+\alpha A X-g(A X, \xi) A \xi
$$

which for $X=U$ and $X=\phi U$ yields

$$
\begin{aligned}
\text { (i) } l U & =\frac{c}{4} U+\alpha A U-\beta A \xi, \\
\text { (ii) } l \phi U & =\frac{c}{4} \phi U+\alpha A \phi U .
\end{aligned}
$$

The scalar products of (17)(i) with $U$ and $\phi U$ yield, respectively,

$$
\begin{gathered}
g(A U, U)=\frac{\gamma}{\alpha}-\frac{c}{4 \alpha}+\frac{\beta^{2}}{\alpha}, \\
g(A U, \phi U)=g(A \phi U, U)=\frac{\delta}{\alpha},
\end{gathered}
$$

where $\gamma=g(l U, U)$ and $\delta=g(l U, \phi U)$. From (18), (19), and $g(A U, \xi)=g(A \xi, U)=\beta$ we obtain $A U=\left(\gamma / \alpha+\beta^{2} / \alpha-\right.$ $c / 4 \alpha) U+\beta \xi$. From (19), $g(A \phi U, \xi)=g(A \xi, \phi U)=0$, and $\epsilon=g(l \phi U, \phi U)$ we obtain $A \phi U=(\epsilon / \alpha-c / 4 \alpha) \phi U+(\delta / \alpha) U$. In order to prove (12) we need to show that $\gamma=\epsilon$ and $\delta=0$. Combining the analysis of $A U$ and $A \phi U$ with (11) and (17) we obtain $l U=\gamma U+\delta \phi U$ and $l \phi U=\delta U+\epsilon \phi U$. The last two equations and $\phi l U-l \phi U=\psi(U) l U$ which holds due to (1) yield

$$
\begin{aligned}
& \text { (i) } \gamma-\epsilon=\psi(U) \delta, \\
& \text { (ii) }-2 \delta=\psi(U) \gamma .
\end{aligned}
$$

Moreover, the decompositions of $l U$ and $l \phi U$ combined with $\phi l \phi U+l U=\psi(\phi U) l \phi U$ which holds due to (1) and (4) yield

$$
\begin{aligned}
\text { (i) } \gamma-\epsilon & =\psi(\phi U) \delta \text {, } \\
\text { (ii) } 2 \delta & =\psi(\phi U) \epsilon .
\end{aligned}
$$

Let us assume that $\delta \neq 0$ in a neighborhood of a point in $\mathcal{N}$. Then (20)(i) and (21)(i) give $\psi(U)=\psi(\phi U)$. Apparently $\psi(U) \gamma \neq 0$; otherwise (20)(ii) would yield $\delta=0$. As a result, (20) and (21) lead, respectively, to $-\gamma(\gamma-\epsilon)=2 \delta^{2}$ and $\epsilon(\gamma-$ $\epsilon)=2 \delta^{2}$. The last two relations are added and result in $(\gamma-$ $\epsilon)^{2}=-4 \delta^{2}$ which is a contradiction. This means that $\delta=0$ holds, and (20) and (21) imply $\gamma=\epsilon$.

Equation (13) is obtained from (12) and relation (6) for $X=\xi, X=U$, and $X=\phi U$. Next we recall the rule

$$
X g(Y, Z)=g\left(\nabla_{X} Y, Z\right)+g\left(Y, \nabla_{X} Z\right) \text {. }
$$

By virtue of (22) for $X=Z=\xi, Y=U$ and for $X=$ $\xi, Y=Z=U$, it is shown, respectively, that $\nabla_{\xi} U \perp \xi$ and $\nabla_{\xi} U \perp U$, which means $\nabla_{\xi} U=\kappa_{1} \phi U$. In a similar way, (22) for $X=Y=Z=U$ and $X=Z=U, Y=\xi$ yields, respectively, $\nabla_{U} U \perp U$ and $\nabla_{U} U \perp \xi$. So $\nabla_{U} U=\kappa_{2} \phi U$ holds. Finally, (22) for $X=\phi U, Y=Z=U$ and $X=\phi U, Y=U, Z=\xi$ (with the aid of (13)) yields, respectively, $\nabla_{\phi U} U \perp U$ and $g\left(\nabla_{\phi U} U, \xi\right)=$ $\gamma / \alpha-c / 4 \alpha$. Therefore we have $\nabla_{\phi U} U=\kappa_{3} \phi U+(\gamma / \alpha-c / 4 \alpha) \xi$ and (14) has been proved. In order to prove (15) we use the second of (6) with the combinations, (i) $X=\xi, Y=U$, (ii) $X=Y=U$, and (iii) $X=\phi U, Y=U$, and make use of (11), (12), and (14).

Lemma 2. Let $M$ be a real hypersurface of a complex projective plane $\mathbb{C} P^{2}$, satisfying (1). Then in $\mathcal{N}$ one has $(\phi U(\gamma / \alpha-$ $c / 4 \alpha))=(3 \beta / \alpha)\left[(\gamma / \alpha-c / 4 \alpha)^{2}-c / 4\right]$.

Proof. Putting $X=U, Y=\xi$ in (8), we obtain $\left(\nabla_{U} A\right) \xi$ $\left(\nabla_{\xi} A\right) U=-(c / 4) \phi U$. Combining the last equation with (11) and Lemma 1, it follows that

$$
\begin{gathered}
{[(U \alpha)-(\xi \beta)] \xi+\left[(U \beta)-\left(\xi\left(\frac{\gamma}{\alpha}-\frac{c}{4 \alpha}+\frac{\beta^{2}}{\alpha}\right)\right)\right] U} \\
+\left[\gamma-\frac{c}{4}+\kappa_{2} \beta-\left(\frac{\gamma}{\alpha}-\frac{c}{4 \alpha}\right)\left(\frac{\gamma}{\alpha}-\frac{c}{4 \alpha}+\frac{\beta^{2}}{\alpha}\right)\right. \\
\left.-\kappa_{1} \frac{\beta^{2}}{\alpha}\right] \phi U=-\frac{c}{4} \phi U .
\end{gathered}
$$


The last equation because of the linear independency of $U$, $\phi U$, and $\xi$ yields

$$
\begin{aligned}
& (U \alpha)=(\xi \beta), \\
& (U \beta)=\left(\xi\left(\frac{\gamma}{\alpha}-\frac{c}{4 \alpha}+\frac{\beta^{2}}{\alpha}\right)\right), \\
& \gamma+\kappa_{2} \beta-\left(\frac{\gamma}{\alpha}-\frac{c}{4 \alpha}\right)\left(\frac{\gamma}{\alpha}-\frac{c}{4 \alpha}+\frac{\beta^{2}}{\alpha}\right)-\kappa_{1} \frac{\beta^{2}}{\alpha}=0 .
\end{aligned}
$$

In the same way, putting $X=\phi U, Y=\xi$ in (8), we obtain $\left(\nabla_{\phi U} A\right) \xi-\left(\nabla_{\xi} A\right) \phi U=(c / 4) U$. Combining the last equation with (11) and Lemma 1, we have

$$
\begin{aligned}
& (\phi U \beta)+\left(\frac{\gamma}{\alpha}-\frac{c}{4 \alpha}\right)\left(\frac{\gamma}{\alpha}-\frac{c}{4 \alpha}+\frac{\beta^{2}}{\alpha}\right)-\kappa_{1} \frac{\beta^{2}}{\alpha}-\beta^{2} \\
& -\gamma=0, \\
& \kappa_{3} \beta=\xi\left(\frac{\gamma}{\alpha}-\frac{c}{4 \alpha}\right), \\
& (\phi U \alpha)+3 \beta\left(\frac{\gamma}{\alpha}-\frac{c}{4 \alpha}\right)-\kappa_{1} \beta-\alpha \beta=0 .
\end{aligned}
$$

Similarly, putting $X=U, Y=\phi U$ in (8), we get $\left(\nabla_{U} A\right) \phi U-$ $\left(\nabla_{\phi U} A\right) U=-(c / 2) \xi$, which, by use of (11) and Lemma 1, implies that

$$
\begin{aligned}
& -\kappa_{2} \frac{\beta^{2}}{\alpha}-3 \beta\left(\frac{\gamma}{\alpha}-\frac{c}{4 \alpha}\right)-\frac{\beta^{3}}{\alpha} \\
& +\left(\phi U\left(\frac{\gamma}{\alpha}-\frac{c}{4 \alpha}+\frac{\beta^{2}}{\alpha}\right)\right)=0, \\
& U\left(\frac{\gamma}{\alpha}-\frac{c}{4 \alpha}\right)=\kappa_{3} \frac{\beta^{2}}{\alpha} .
\end{aligned}
$$

We expand (30) and then replace the terms $\kappa_{2},(\phi U \beta)$, and $(\phi U \alpha)$ from (26), (27), and (29), respectively. The final equation is

$$
\left(\phi U\left(\frac{\gamma}{\alpha}-\frac{c}{4 \alpha}\right)\right)=\frac{3 \beta}{\alpha}\left[\left(\frac{\gamma}{\alpha}-\frac{c}{4 \alpha}\right)^{2}-\frac{c}{4}\right] .
$$

Lemma 3. Let $M$ be a real hypersurface of a complex projective plane $\mathbb{C} P^{2}$, satisfying (1). Then, $\kappa_{3}=0$ holds in $\mathcal{N}$.

Proof. Because of (14), (15), (28), (31), and Lemma 2, the wellknown relation $[U, \phi U]=\nabla_{U} \phi U-\nabla_{\phi U} U$ takes the form

$$
\begin{aligned}
{[U, \phi U]\left(\frac{\gamma}{\alpha}-\frac{c}{4 \alpha}\right)=} & -\frac{\kappa_{2} \kappa_{3} \beta^{2}}{\alpha} \\
& -\kappa_{3} \beta\left(\frac{\gamma}{\alpha}-\frac{c}{4 \alpha}+\frac{\beta^{2}}{\alpha}\right) \\
& -\frac{3 \beta \kappa_{3}}{\alpha}\left[\left(\frac{\gamma}{\alpha}-\frac{c}{4 \alpha}\right)^{2}-\frac{c}{4}\right] \\
& -\kappa_{3} \beta\left(\frac{\gamma}{\alpha}-\frac{c}{4 \alpha}\right) .
\end{aligned}
$$

On the other hand (27), (29), (31), and Lemma 2 yield

$$
\begin{aligned}
{[U, \phi U] } & \left(\frac{\gamma}{\alpha}-\frac{c}{4 \alpha}\right) \\
= & U\left(\phi U\left(\frac{\gamma}{\alpha}-\frac{c}{4 \alpha}\right)\right)-\phi U\left(U\left(\frac{\gamma}{\alpha}-\frac{c}{4 \alpha}\right)\right) \\
= & \frac{3(U \beta)}{\alpha}\left[\left(\frac{\gamma}{\alpha}-\frac{c}{4 \alpha}\right)^{2}-\frac{c}{4}\right] \\
& -\frac{3 \beta(U \alpha)}{\alpha^{2}}\left[\left(\frac{\gamma}{\alpha}-\frac{c}{4 \alpha}\right)^{2}-\frac{c}{4}\right] \\
& +\frac{6 \kappa_{3} \beta^{3}}{\alpha^{2}}\left(\frac{\gamma}{\alpha}-\frac{c}{4 \alpha}\right)-\frac{\beta^{2}}{\alpha}\left(\phi U\left(\kappa_{3}\right)\right) \\
& +\frac{2 \kappa_{3} \beta}{\alpha}\left(\frac{\gamma}{\alpha}-\frac{c}{4 \alpha}\right)\left(\frac{\gamma}{\alpha}-\frac{c}{4 \alpha}+\frac{\beta^{2}}{\alpha}\right)-\frac{2 \kappa_{3} \beta \gamma}{\alpha} \\
& -\frac{\kappa_{1} \kappa_{3} \beta^{3}}{\alpha^{2}}-\frac{\kappa_{3} \beta^{3}}{\alpha}-\frac{3 \kappa_{3} \beta^{3} \gamma}{\alpha^{3}}+\frac{3 \kappa_{3} c \beta^{3}}{4 \alpha^{3}} .
\end{aligned}
$$

The last equations using (24), (25), and (28) yield

$$
\begin{gathered}
\frac{3}{\alpha}\left[\left(\frac{\gamma}{\alpha}-\frac{c}{4 \alpha}\right)^{2}-\frac{c}{4}\right](\xi \beta)-\frac{3 \beta}{\alpha^{2}}\left[\left(\frac{\gamma}{\alpha}-\frac{c}{4 \alpha}\right)^{2}-\frac{c}{4}\right] \\
\cdot(\xi \alpha)-\beta\left(\phi U \kappa_{3}\right)=\left[2 c-\beta \kappa_{2}+\frac{\beta^{2}}{\alpha} \kappa_{1}\right. \\
\left.-8\left(\frac{\gamma}{\alpha}-\frac{c}{4 \alpha}\right)^{2}-\frac{5 \beta^{2}}{\alpha}\left(\frac{\gamma}{\alpha}-\frac{c}{4 \alpha}\right)\right] \kappa_{3} .
\end{gathered}
$$

Following a similar way, we calculate $[\xi, \phi U](\gamma / \alpha-$ $c / 4 \alpha)=\left(\nabla_{\xi} \phi U-\nabla_{\phi U} \xi\right)(\gamma / \alpha-c / 4 \alpha)$ and then $[\xi, \phi U](\gamma / \alpha-$ $c / 4 \alpha)=\xi(\phi U(\gamma / \alpha-c / 4 \alpha))-\phi U(\xi(\gamma / \alpha-c / 4 \alpha))$. By equalizing the results we obtain

$$
\begin{aligned}
\frac{3}{\alpha}[ & \left.\left(\frac{\gamma}{\alpha}-\frac{c}{4 \alpha}\right)^{2}-\frac{c}{4}\right](\xi \beta) \\
& -\frac{3 \beta}{\alpha^{2}}\left[\left(\frac{\gamma}{\alpha}-\frac{c}{4 \alpha}\right)^{2}-\frac{c}{4}\right](\xi \alpha)-\beta\left(\phi U \kappa_{3}\right) \\
= & {\left[\gamma-\left(\frac{\gamma}{\alpha}-\frac{c}{4 \alpha}\right)^{2}-\frac{6 \beta^{2}}{\alpha}\left(\frac{\gamma}{\alpha}-\frac{c}{4 \alpha}\right)\right] \kappa_{3} . }
\end{aligned}
$$
obtain

Comparing (35) with (36) and by making use of (26) we

$$
\kappa_{3}\left[\left(\frac{\gamma}{\alpha}-\frac{c}{4 \alpha}\right)^{2}-\frac{c}{4}\right]=0
$$

Due to (32), we have $\gamma \neq c / 4$. So if we had $\kappa_{3} \neq 0$ in neighborhood of point in $\mathcal{N}$ then (37) would imply $(\gamma / \alpha-$ $c / 4 \alpha)^{2}=c / 4$. By differentiating the last relation with respect to $\xi$, in combination with $\gamma \neq c / 4$ and (28), we would obtain $\kappa_{3}=0$ which is a contradiction.

Therefore $\kappa_{3}$ holds in $\mathcal{N}$. 


\section{Proof of Main Theorem}

We first prove the following proposition.

Proposition 4. Let $M$ be a real hypersurface of a complex projective plane $\mathbb{C} P^{2}(c \neq 0)$, satisfying $(1)$ and $\left(\nabla_{\xi} \xi \alpha\right)=0$. Then $M$ is Hopf.

Proof. We keep working in $\mathcal{N}$. By virtue of Lemma 3 and (11), (28), and (31), we obtain $[A \xi, \xi](\gamma / \alpha-c / 4 \alpha)=A \xi(\xi(\gamma / \alpha-$ $c / 4 \alpha))-\xi(A \xi(\gamma / \alpha-c / 4 \alpha))=0$. However, from Lemmas 1,2 , and 3 and (11) we calculate $[A \xi, \xi](\gamma / \alpha-c / 4 \alpha)=$ $(3 \beta / \alpha)\left(\nabla_{A \xi} \xi-\nabla_{\xi} A \xi\right)(\gamma / \alpha-c / 4 \alpha)=\left(\gamma / \alpha-c / 4 \alpha+\beta^{2} / \alpha-\right.$ $\left.\kappa_{1}\right)\left((\gamma / \alpha-c / 4 \alpha)^{2}-c / 4\right)$. The two expressions of $[A \xi, \xi](\gamma / \alpha-$ $c / 4 \alpha)$ yield

$$
\left(\frac{\gamma}{\alpha}-\frac{c}{4 \alpha}+\frac{\beta^{2}}{\alpha}-\kappa_{1}\right)\left(\left(\frac{\gamma}{\alpha}-\frac{c}{4 \alpha}\right)^{2}-\frac{c}{4}\right)=0 .
$$

Let us assume that there exists a point in $\mathcal{N}$, such that $(\gamma / \alpha-c / 4 \alpha)^{2}-c / 4 \neq 0$ in a neighborhood of this point. Then (38) implies

$$
\kappa_{1}=\frac{\gamma}{\alpha}-\frac{c}{4 \alpha}+\frac{\beta^{2}}{\alpha} .
$$

Based on the definition of the vector field $U$ in Section 2, condition $\left(\nabla_{\xi} \xi \alpha\right)=0$ is equivalent to $\phi U \alpha=0$. The last relation, (39), and (29) yield

$$
\gamma-\frac{c}{4}=\frac{\alpha^{2}+\beta^{2}}{2}
$$

By virtue of (39) and (40) we simplify (27) and obtain

$$
\phi U \beta=\frac{3 \beta^{4}}{4 \alpha^{2}}+\beta^{2}+\frac{\alpha^{2}}{4}+\frac{c}{4} .
$$

We modify (32) by replacing the term $\gamma / \alpha-c / 4 \alpha$ from (40). Then we make calculations with the aid of $(41),(\phi U \alpha)=0$, to obtain $\beta^{2}+\alpha^{2}=2 c$. The last equation is differentiated with respect to $\phi U$, giving $(\phi U \beta)=0$. As a result, (41) yields $c<0$ which is a contradiction and $(\gamma / \alpha-c / 4 \alpha)^{2}=c / 4$ holds in $\mathcal{N}$.

Combining $(\phi U \alpha)=0$ and (29) we acquire

$$
\kappa_{1}=3\left(\frac{\gamma}{\alpha}-\frac{c}{4 \alpha}\right)-\alpha .
$$

The combination of (26) and (42) leads to

$$
\kappa_{2} \beta=4 \frac{\beta^{2}}{\alpha}\left(\frac{\gamma}{\alpha}-\frac{c}{4 \alpha}\right)-\left(\gamma-\frac{c}{4}\right)-\beta^{2} .
$$

In addition, (27), $(\gamma / \alpha-c / 4 \alpha)^{2}=c / 4$, and (42) yield

$$
(\phi U \beta)=\left(\frac{\gamma}{\alpha}-\frac{c}{4 \alpha}\right)\left(\frac{2 \beta^{2}+\alpha^{2}}{\alpha}\right) .
$$

We estimate the vector field $R(\phi U, \xi) U$ from (7) and Lemma 1 as $R(\phi U, \xi) U=\beta(\gamma / \alpha-c / 4 \alpha) \phi U$. The same vector field is calculated from $R(\phi U, \xi) U=\nabla_{\phi U} \nabla_{\xi} U-\nabla_{\xi} \nabla_{\phi U} U-$ $\nabla_{[\phi U, \xi]} U,(42),(\gamma / \alpha-c / 4 \alpha)^{2}=c / 4$, and $(\phi U \alpha)=0:$ $R(\phi U, \xi) U=-4 \beta(\gamma / \alpha-c / 4 \alpha) \phi U-2(\gamma / \alpha-c / 4 \alpha) \kappa_{2} \phi U+$ $\alpha \kappa_{2} \phi U+\alpha \beta$. The two expressions of $R(\phi U, \xi) U$ are equalized and the outcome is modified as follows: first we multiply with $\beta$, then we replace the term $\kappa_{2} \beta$ from (43), and finally we divide with $\gamma-c / 4$. After these steps, we are led to

$$
\frac{\beta^{2}}{\alpha}-8 \frac{\beta^{2}}{\alpha^{2}}\left(\frac{\gamma}{\alpha}-\frac{c}{4 \alpha}\right)+2\left(\frac{\gamma}{\alpha}-\frac{c}{4 \alpha}\right)-\alpha=0 .
$$

We differentiate the above relation and utilize $(\phi U \beta) \neq 0$ (otherwise (44) yields $c<0$ which is a contradiction) in order to get $\gamma / \alpha-c / 4 \alpha=\alpha / 8$. The last two equations give $\alpha=0$ which is a contradiction. Therefore we have a contradiction in $\mathcal{N}$; hence $\mathcal{N}=\emptyset$ and $M$ is Hopf.

From Proposition 4 we have on $M$

$$
A \xi=\alpha \xi, \quad \alpha=g(A \xi, \xi)
$$

and $\alpha$ is a constant [5]. We consider $\phi$-basis $\{e, \phi e, \xi\}$ which satisfies

$$
\begin{gathered}
A e=\lambda_{1} e, \\
A \phi e=\lambda_{2} \phi e, \\
A \xi=\alpha \xi .
\end{gathered}
$$

From (7) and (47) we obtain

$$
\begin{aligned}
l e & =\frac{c}{4} e+\alpha \lambda_{1} e, \\
l \phi e & =\frac{c}{4} \phi e+\alpha \lambda_{2} \phi e .
\end{aligned}
$$

By making use of (1) with $X=e$, in combination with (48), we obtain

$$
\alpha\left(\lambda_{1}-\lambda_{2}\right)=0
$$

If $\alpha \neq 0$ then $\lambda_{1}=\lambda_{2}=\lambda$ and $\lambda$ is the root of the quadratic $t^{2}-\alpha t-c / 4$ [5] and consequently a constant. The classification follows from [3]. Since $M$ is of type $A$ then (due to [5]) $M$ satisfies $\phi A=A \phi$. The last condition and (1) and (15) lead to $\psi(X) l X=0, \forall X \in \mathbb{D}$, which is combined with $l \xi=0$, resulting in $\psi(X) l X=0, \forall X \in T_{p} M=0$.

\section{Competing Interests}

The author declares that there are no competing interests.

\section{References}

[1] R. Takagi, "On homogeneous real hypersurfaces in a complex projective space," Osaka Journal of Mathematics, vol. 10, pp. 495-506, 1973.

[2] R. Takagi, "Real hypersurfaces in a complex projective space with constant principal curvatures," Journal of the Mathematical Society of Japan, vol. 27, pp. 43-53, 1975. 
[3] M. Kimura, "Real hypersurfaces and complex submanifolds in complex projective space," Transactions of the American Mathematical Society, vol. 296, no. 1, pp. 137-149, 1986.

[4] J. Berndt, "Real hypersurfaces with constant principal curvatures in complex hyperbolic space," Journal für die Reine und Angewandte Mathematik, vol. 395, pp. 132-141, 1989.

[5] R. Niebergall and P. J. Ryan, Real Hypersurfaces in Complex Space Forms, vol. 32 of Mathematical Sciences Research Institute Publications, Cambridge University Press, Cambridge, UK, 1997.

[6] J. T. Cho and U.-H. Ki, "Real hypersurfaces in complex space forms with Reeb flow symmetric structure Jacobi operator," The Canadian Mathematical Bulletin, vol. 51, no. 3, pp. 359-371, 2008.

[7] T. A. Ivey and P. J. Ryan, "The structure Jacobi operator for real hypersurfaces in $\mathbb{C P}^{2}$ and $\mathbb{C} H^{2}$," Results in Mathematics, vol. 56, pp. $473-488,2009$.

[8] U.-H. Ki, J. D. Dios Perez, F. G. Santos, and Y. J. Suh, "Real hypersurfaces in complex space forms with $\varepsilon$-parallel Ricci tensor and structure Jacobi operator," Journal of the Korean Mathematical Society, vol. 44, no. 2, pp. 307-326, 2007.

[9] Th. Theofanidis, "Real hypersurfaces with pseudo- $\mathbb{D}$-parallel structure Jacobi operator in complex hyperbolic spaces," Colloquium Mathematicum, vol. 134, no. 1, pp. 93-112, 2014.

[10] J. T. Cho and U.-H. Ki, "Real hypersurfaces of a complex projective space in terms of the Jacobi operators," Acta Mathematica Hungarica, vol. 80, no. 1-2, pp. 155-167, 1998.

[11] H. Ki, "The Ricci tensor and the structure Jacobi operator of real hypersurfaces in complex space forms," in Proceedings of the 9th International Workshop on Differential Geometry, pp. 8596, Kyungpook National University, Taegu, Republic of Korea, 2005.

[12] U.-H. Ki, S. J. Kim, and S.-B. Lee, “The structure Jacobi operator on real hypersurfaces in a nonflat complex space form," Bulletin of the Korean Mathematical Society, vol. 42, no. 2, pp. 337-358, 2005.

[13] U.-H. Ki, A.-A. Lee, and S.-B. Lee, "On real hypersurfaces of a complex space form in terms of Jacobi operators," Communications of the Korean Mathematical Society, vol. 13, no. 2, pp. 317336, 1998.

[14] U.-H. Ki, S. Nagai, and R. Takagi, "Real hypersurfaces in non-flat complex space forms concerned with the structure Jacobi operator and Ricci tensor," in Topics in Almost Hermitian Geometry and Related Fields, pp. 140-156, World Scientific, Hackensack, NJ, USA, 2005.

[15] T. Theofanidis and Ph. J. Xenos, "Real hypersurfaces of non-flat complex space forms in terms of the Jacobi structure operator," Publicationes Mathematicae Debrecen, vol. 87, no. 1-2, pp. 175189, 2015.

[16] T. E. Cecil and P. J. Ryan, "Focal sets and real hypersurfaces in complex projective space," Transactions of the American Mathematical Society, vol. 269, no. 2, pp. 481-499, 1982.

[17] D. E. Blair, Riemannian Geometry of Contact and Symplectic Manifolds, vol. 203 of Progress in Mathematics, Birkhäuser, 2002. 


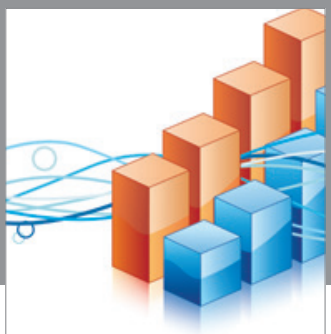

Advances in

Operations Research

vatem alat4

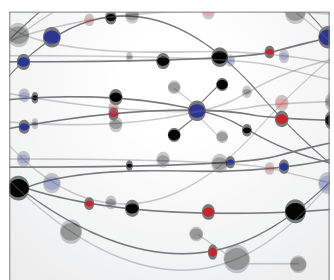

\section{The Scientific} World Journal
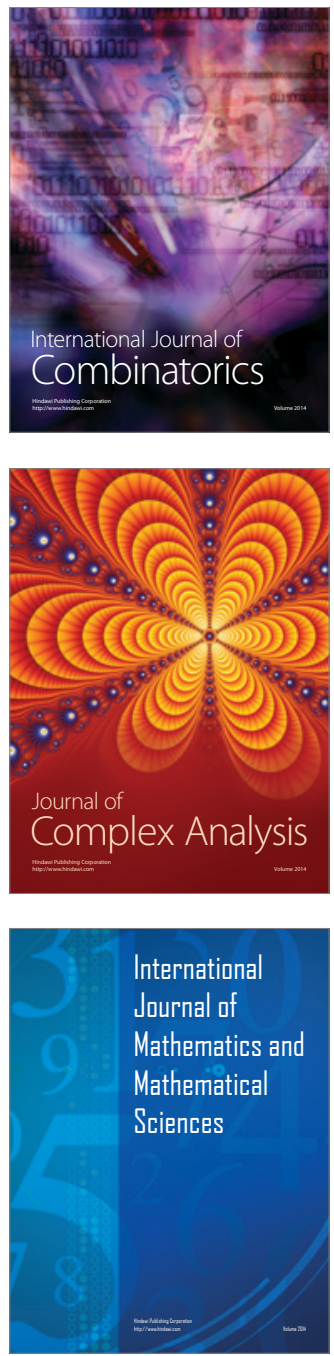
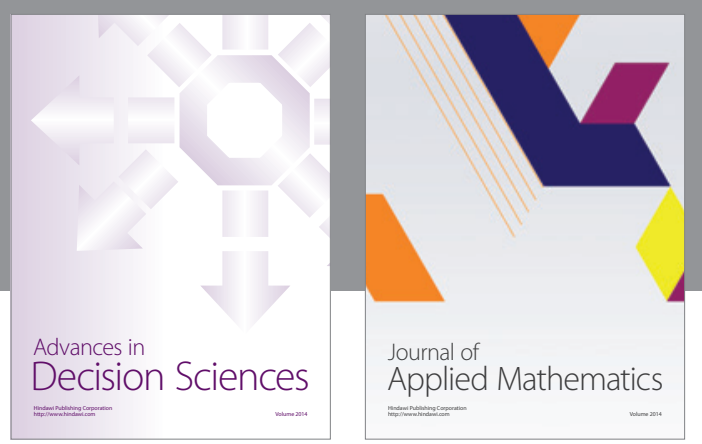

Algebra

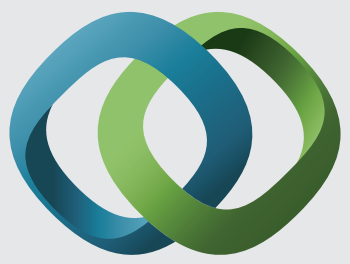

\section{Hindawi}

Submit your manuscripts at

http://www.hindawi.com
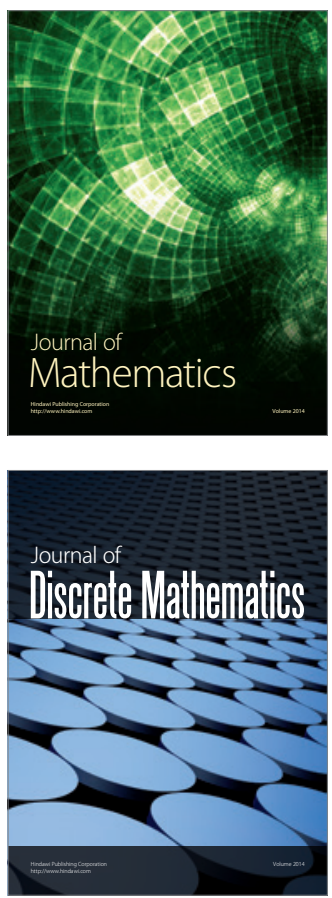

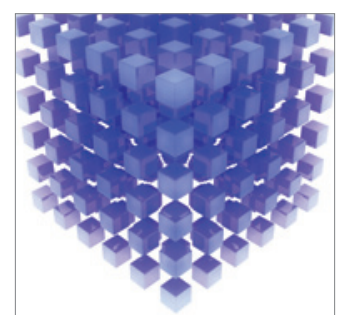

Mathematical Problems in Engineering
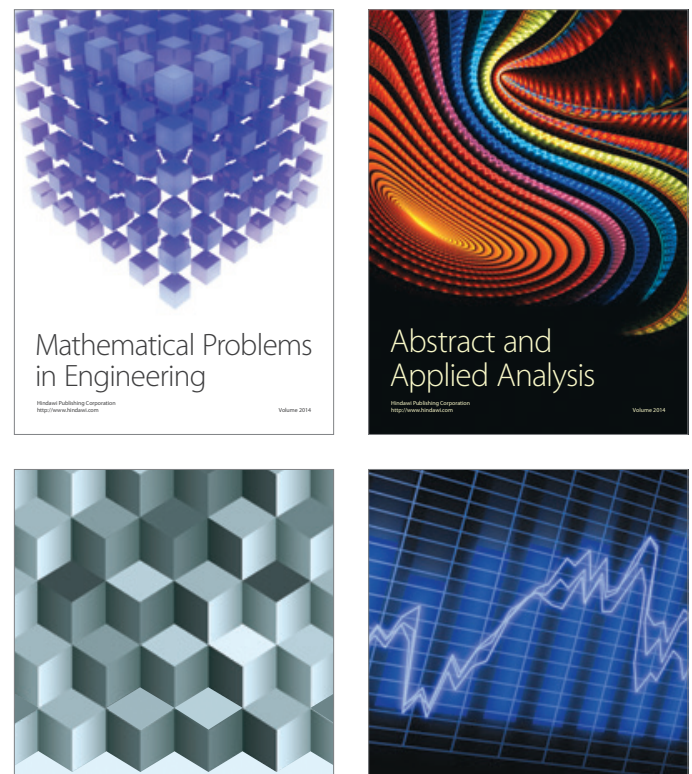

Journal of

Function Spaces

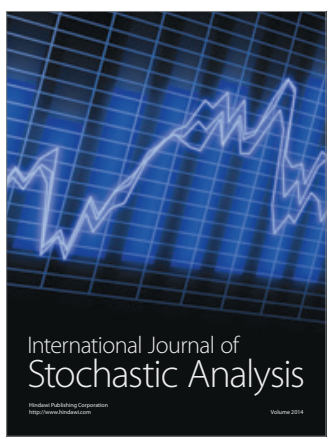

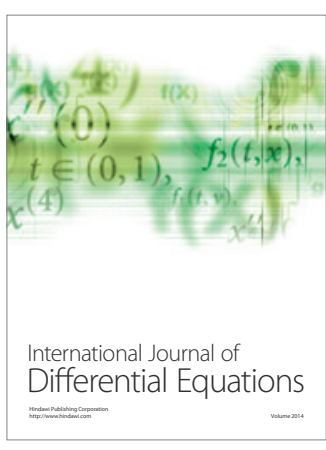
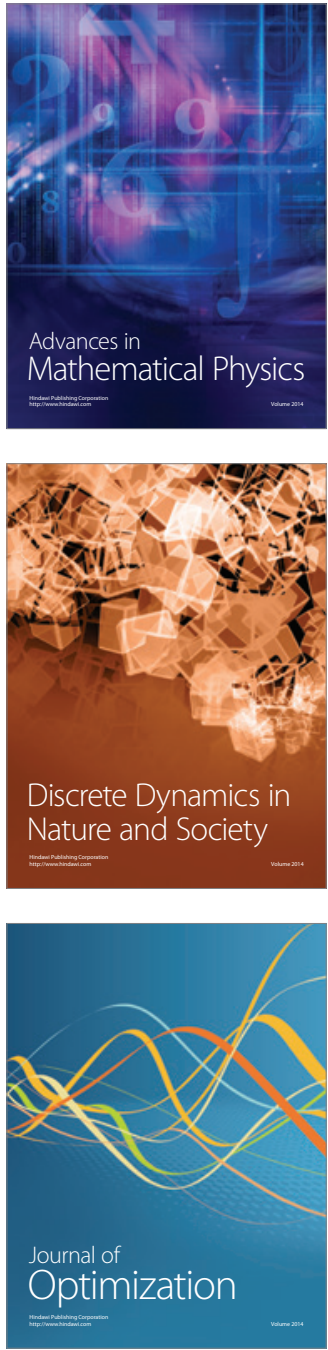